\title{
Evaluación de la medulación de fibras de lanas y fibras especiales de algunas especies de animales
}

\author{
Evaluation of wool and luxury fiber medullation of some animal species \\ Giancarlo Berolatti Obando ${ }^{1}$, Lusmenia Ruiz Mejía ${ }^{2}$, Flor Alicia Cabrera \\ Bustamante$^{2}$, Jorge Aliaga Gutiérrez ${ }^{3}$, Max David Quispe Bonilla ${ }^{4}$, \\ Edgar Carlos Quispe Peña ${ }^{3,5,6}$
}

\section{Resumen}

Existen argumentos a favor de la reducción de la incidencia de medulación, a fin de realizar la mejora de las fibras desde el punto de vista de la producción y procesamiento textil, por lo que se requiere reforzar el conocimiento en torno a esta característica. Por tanto, el presente trabajo tuvo como objetivo evaluar la incidencia y relación entre los tipos de medulación, así como determinar su relación con la finura en fibras de ovino, alpaca, llama, paco-vicuña y conejo Angora. Se tomaron muestras de lana y fibra ( $n=15$ por especie animal) y adicionalmente 140 muestras de alpacas tuis macho de color blanco. Se determinó la media del diámetro de fibra (MDF) y los porcentajes de fibras meduladas $(\% \mathrm{FM})$ y no meduladas $(\% \mathrm{FNM})$, y en esta última se determinaron los porcentajes de fibras de medulación fragmentada, discontinua, continua y fuertemente medulada o tipo kemp. Los resultados obtenidos mostraron que las fibras de lana Merino presentaron pocas fibras meduladas, ocurriendo lo contrario en fibras de conejo Angora, mientras que las fibras de alpacas tuis, llamas y paco-vicuñas tuvieron porcentajes de

\footnotetext{
${ }^{1}$ Universidad Nacional de Juliaca, Puno, Perú

${ }^{2}$ Escuela Profesional de Agroindustrias, Universidad Nacional Autónoma de Chota, Perú

${ }^{3}$ Departamento de Producción Animal, Facultad de Zootecnia, Universidad Nacional Agraria La Molina, Lima, Perú

${ }^{4}$ Laboratorio de Desarrollo e Innovaciones Tecnológicas, Maxcorp Technologies SAC, Lima, Perú

${ }^{5}$ Centro de Investigación Científica y Desarrollo Tecnológico, Natural Fiber's Tech SAC, Lima, Perú

${ }^{6}$ E-mail: edgarquispe62@gmail.com
}

Recibido: 14 de abril de 2021

Aceptado para publicación: 23 de septiembre de 2021

Publicado: 27 de octubre de 2021

CLos autores. Este artículo es publicado por la Rev Inv Vet Perú de la Facultad de Medicina Veterinaria, Universidad Nacional Mayor de San Marcos. Este es un artículo de acceso abierto, distribuido bajo los términos de la licencia Creative Commons Atribución 4.0 Internacional (CC BY 4.0) [https:// creativecommons.org/licenses/by/4.0/deed.es] que permite el uso, distribución y reproducción en cualquier medio, siempre que la obra original sea debidamente citada de su fuente original 
medulación moderados (22-40\%), encontrándose médulas aún en fibras muy finas. Se determinó una relación directa entre la MDF y el \%FM en todos los tipos de fibras, a excepción de la lana de ovino. Se concluye que el \%FM es una característica que debería disminuirse en llamas y alpacas y, por tanto, debiera ser considerado como criterio de selección en los programas de mejoramiento genético, pero solo en torno a reducir drásticamente la incidencia de fibras fuertemente meduladas.

Palabras clave: fibras, camélidos sudamericanos, pelo de conejo, kemp, medulación fragmentada

\section{Abstract}

There are arguments in favour of reducing the incidence of medullation to improve fibres quality from the point of view of textile production and processing, and therefore it is necessary to reinforce the knowledge of this characteristic. Based on that, this study aimed to evaluate the incidence and relationship between the types of medullation, as well as to determine its relationship with the fineness in fibres of sheep, alpaca, llama, paco-vicuña and Angora rabbit. Samples of wool and fibre ( $\mathrm{n}=15$ per animal species) and additionally 140 samples of white male tuis alpacas were collected. The mean fibre diameter (MFD) and the percentages of medullated (\% MF) and non-medullated (\% NMF) fibres were determined, and in the latter, the percentages of fibres with fragmented, discontinuous, continuous and strongly medullated or kemp-type medullation were determined. Results showed that Merino wool fibres presented few medullary fibres, the opposite occurred in Angora rabbit fibres, while the fibres of alpaca tuis, llamas and paco-vicuñas had moderate percentages of medullation (22-40\%), with medullary still found in very high fine fibres. A direct relationship between MFD and \%MF was determined in all types of fibres, except for sheep wool. It is concluded that the $\% \mathrm{MF}$ is a characteristic that should be diminished in llamas and alpacas and, therefore, it should be considered as a selection criterion in genetic improvement programmes, but only to reduce the incidence of strongly medullated fibres.

Key words: fibres, South American Camelids, rabbit hair, kemp, fragmented medullation

\section{INTRODUCCIÓN}

Aunque la finura de la fibra es la principal característica que define la calidad de la lana y fibra en los diferentes procesos de producción y transformación, la presencia y el porcentaje de medulación también son importantes (Gupta et al., 1981; McGregor, 2006), porque determinan su uso en la industria e influyen en el grado de calidad de la fibra, hilos y tejidos, afectando también su apariencia y rendimiento.
Las fibras meduladas se caracterizan por tener un canal central (médula) conteniendo residuos celulares y bolsas de aire que se dirigen en forma continua o fragmentada a lo largo de la fibra (Gupta et al., 1981; Hunter, 1993; Lupton y Pfeiffer, 1998; McGregor et al., 2013); no obstante, resulta de particular interés las fibras fuertemente meduladas (fibras objetables o tipo kemp), donde la médula es mayor de $60 \%$ del diámetro de la fibra (ASTM, 1986). 
Las fibras meduladas, principalmente las fibras objetables, constituyen un defecto desde el punto de vista textil, debido al efecto sobre las propiedades del teñido en los tejidos, pues las bolsas de aire presentes en la médula les dan una apariencia más clara que las fibras normales, por afectar las propiedades ópticas de la luz que pasa a través de la fibra por difracción y no por la diferencia de consumo de tinte por la queratina de la fibra (McGregor, 2002). También son menos resistentes, aunque más elásticas, y al parecer están asociados a un mayor grosor y pérdida de rizos (Gupta et al., 1981; Moore, 2015), por lo que tienen efectos adversos sobre la suavidad, vellosidad, rigidez y el picor de hilos y telas, siendo así que muchos consideran a las fibras meduladas como contaminantes de los vellones de lana, mohair, cachemira, alpaca y llama (Hunter, 1993; Balasingam, 2005; Frank et al., 2009; McGregor, 2012; Cruz et al., 2019).

A través del tiempo se han realizado enormes esfuerzos para disminuir la MDF de las fibras de lana y alpacas. En el caso de Perú, las alpacas llegan a tener medias de diámetro de fibra entre 20 y $23 \mu \mathrm{m}$ (Montes et al., 2008; Quispe et al., 2009; Vásquez et al., 2015, Omachea et al., 2015; Pinares et al., 2018; Cruz et al., 2019), que a la clasificación se encuentran dentro de la calidad Alpaca Baby (INDECOPI, 2014). Los programas de mejoramiento genético no consideran el porcentaje de medulación de las fibras; sin embargo, al tener una heredabilidad moderada, posibilita su uso como criterio de selección, lo que permitiría disminuir el efecto del factor de picazón ocasionado principalmente por fibras fuertemente meduladas (Pinares et al., 2018; Cruz et al., 2019), pero también se lograría afinar aún más la fibra. En este sentido, McGregor (2006) y Onal et al. (2007) refieren que la correlación entre medulación y diámetro de la fibra, en alpacas, llamas y conejos entre otros, es positiva con valores entre moderado a alto.
La medulación no siempre resulta ser un defecto, pues la presencia de médula torna las fibras más livianas, razón por la cual, tejidos en base a fibras de conejo y alpaca son más livianas que los de lana (Czaplicki, 2012) y brindan un mejor aislamiento térmico (Moore, 2015), debido a la presencia de bolsas de aire que amortigua el intercambio térmico (Czaplicki, 2012). Por tanto, siendo las fibras objetables la causa del problema del picazón de fibras de alpacas, resulta de importancia reducir o eliminar este tipo de fibras, además de las que tienen medulación continua (Pinares et al., 2018).

La incidencia de medulación en lanas de ovinos Merino es muy baja, en fibras de mohair y cachemira es de baja a moderada, en fibras del buey almizclero es moderada (McGregor, 2012), en fibras de llamas es generalmente alta (Martínez et al., 1997; Cochi, 1999; Córdova, 2015; Poma, 2018), en fibras de alpacas varía entre regular y alta (McGregor, 2006; Lupton et al., 2006; Contreras, 2010; Ferguson et al., 2012; Pinares et al., 2018, 2019; Radzik et al., 2018; Cruz et al., 2019), mientras que en pelos de conejo Angora es extremadamente alta (McGregor, 2012) a pesar de que la finura varía entre 10 a $15 \mu \mathrm{m}$ (Chattopadhyay et al., 2005), demostrándose que la incidencia de medulación se incrementa con la edad (Martínez et al., 1997; Lupton et al., 2006, Pinares et al., 2019)

Investigaciones sobre medulación se han realizado con bastante profundidad en lanas y fibras de mohair, utilizando el OFDA 100 y el microscopio de proyección (Balasingam, 2005); sin embargo, en fibras de otras especies existe limitada información al respecto, ya que su determinación mediante el microscopio de proyección resulta bastante laborioso, necesita mucho entrenamiento y los resultados varían de persona a persona (Balasingam, 2005; Shakyawar et al., 2013), mientras que utilizando el OFDA 100 se ob- 
tienen resultados dentro de un rango bastante amplio (Pinares et al., 2019) y en algunos casos discordantes con la realidad, pues al parecer los umbrales de opacidad debieran ser redefinidos para su uso en otros tipos de fibra, diferentes al de la lana o mohair. En esta línea, Rafat et al. (2007) y Pinares et al. (2018) muestran resultados discordantes de medulación medidos con el microscopio de proyección y el OFDA 100 . Por tanto, los reportes sobre tasa de medulación en alpacas y llamas, realizados por McGregor (2006), Lupton et al. (2006), Ferguson et al. (2012) y Cruz et al. (2019, entre otros, deberían ser discutidos.

Estas razones condujeron a realizar el presente trabajo de investigación con la finalidad de evaluar la incidencia y relación entre los diferentes tipos de medulación (no meduladas, con medulación continua, fragmentada, discontinua y fuertemente meduladas), así como su relación con la finura de las fibras de diferentes especies: ovino, alpaca, llama, paco-vicuña y conejo Angora, utilizando mediciones directas y objetivas.

\section{Materiales y Métodos}

Las muestras de lana de ovinos Merino $(n=15)$ y paco vicuña $(n=15)$ fueron obtenidos de dos granjas de Missouri, EEUU, de hembras de variada edad; las muestras de fibras de conejos Angora ( $\mathrm{n}=15)$ se obtuvieron de granjas de pequeños criadores del distrito de Chota, Perú; y las muestras de llamas $(n=15)$ y alpacas $(n=15)$ de machos $y$ hembras adultas (2-7 años) fueron obtenidos del Centro de Investigación y Producción Lachocc, perteneciente a la Universidad Nacional de Huancavelica. Adicionalmente, 140 muestras de fibras de alpacas tuis machos de color blanco fueron obtenidos de granjas de la región Puno, Perú.

Las muestras de fibras de lana de ovino, conejos, alpacas, llamas y paco vicuñas (75 muestras) fueron analizadas en el Labo-

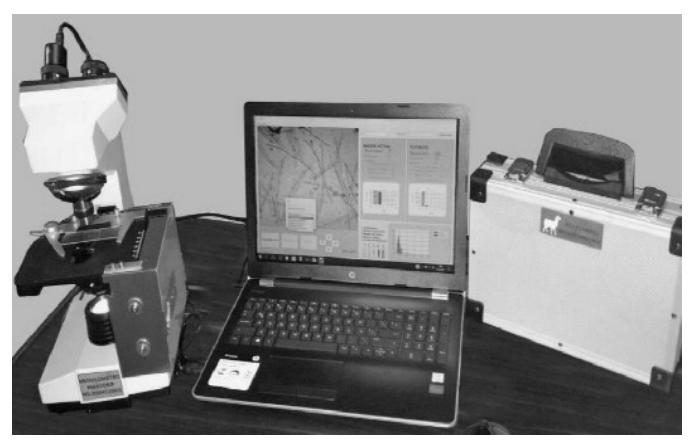

Figura 1. Equipo denominado Medulómetro utilizado para medir el diámetro y el tipo de medulación de muestras de fibras de diversas especies de animales.

ratorio de Fibras Textiles de la Universidad Nacional Autónoma de Chota (Perú); mientras que, las 140 muestras de fibras de alpacas tuis machos fueron analizadas en el Laboratorio de Fibras de la Universidad Nacional de Juliaca. Para el análisis se utilizó el equipo denominado Medulómetro, que evalúa la calidad de la fibra animal, teniendo en cuenta el tipo de medulación (tipo kemp, continua, discontinua, fragmentada y no medulada). El Medulómetro es un microscopio de proyección modificado, al que se incorporaron dos motores y una tarjeta electrónica para el escaneo automatizado, conectado a una computadora que tiene instalado un software propietario (Figura 1).

La norma IWTO-8-04 fue utilizada para la evaluación del diámetro y medulación, para lo cual las fibras fueron cortadas en fragmentos de $0.8 \mathrm{~mm}$ utilizando el micrótomo de Hardy, que luego fueron dispersadas en una lámina portaobjeto utilizando una bagueta y aceite de cedro, y cubierta con una lámina cubreobjetos. Esta preparación fue colocada en el Medulómetro, iniciando las mediciones desde la esquina inferior izquierda para ir avanzando hacia arriba y hacia la derecha. El total de mediciones por muestra fue de $600.75 \pm 7.48$. 
La caracterización de la medulación y diámetro de fibra en las cinco especies estuvo basada en estadística descriptiva (promedio, desviación y error estándar, valores mínimo y máximo). Para las comparaciones entre tasas de medulación se utilizó un modelo lineal general considerando a las especies como variables independientes y como variables dependientes al porcentaje de medulación y la media del diámetro de fibra. Para la evaluación del porcentaje de medulación al no tener una distribución normal se hizo una transformación logarítmica de la proporción de medulación a la que se sumó 1/6. Adicionalmente, para determinar la relación entre medulación y diámetro de fibra se hizo uso de la correlación de Pearson (r) y análisis de regresión simple, realizándose la evaluación de supuestos en base a los residuos (distribución normal, homogeneidad de varianza e independencia de errores) mediante estadísticos y diagramas. El software utilizado fue R v. 3.6.1 (R Core Team, 2019).

\section{Resultados}

\section{Medulación y Diámetro de fibra}

No se encontraron muestras conteniendo médulas tipo kemp en lana de ovinos Merino, pero la tercera parte de ellas presentaban algún otro tipo de medulación. Sin em- bargo, en todas las muestras de fibras de las otras especies (llama, alpaca, conejo, pacovicuña y conejo Angora) se encontraron fibras con algún tipo de medulación, a excepción de una sola muestra de fibra de llama que no contenía fibras meduladas. Asimismo, en más del $53 \%$ de las muestras de camélidos se observaron fibras fuertemente meduladas, tipo «kemp» o fuertemente meduladas. La menor frecuencia (33\%) fue en fibras de conejo Angora (Cuadro 1).

Las fibras de conejo Angora tuvieron la mayor incidencia de medulación, seguido de las fibras de alpacas y llamas, mientras que la menor incidencia se observó en lana de ovino Merino, siendo evidente el efecto de la especie animal sobre el porcentaje de medulación (Cuadro 2). Los menores rangos de variación de la incidencia de medulación se registró en lana de ovino Merino (1.92\%), seguido de la fibra de paco-vicuña (31.8\%) y de conejo Angora (33.17\%); mientras que, los mayores rangos se observaron en fibras de llama ( $85.43 \%$ ), seguido muy de cerca en fibras alpacas tuis machos de color blanco (76.4\%).

Los resultados de la incidencia de medulación y media del diámetro de fibra (MDF) por tipo de medulación se presentan en el Cuadro 3. Las fibras de paco-vicuña no solo son las más finas, sino que también tie-

Cuadro 1. Cantidad y porcentaje de muestras de lanas y fibras que presentan medulación y específicamente, medulación tipo kemp, en diferentes especies animales

\begin{tabular}{lccccc}
\hline \multirow{2}{*}{ Especies } & $\mathrm{n}$ & \multicolumn{2}{c}{ Muestras con medulación } & $\begin{array}{c}\text { Muestras con medulación } \\
\text { tipo kemp }\end{array}$ \\
\cline { 3 - 6 } & & $\mathrm{n}$ & $\%$ & $\mathrm{n}$ & $\%$ \\
\hline Ovino & 15 & 5 & 33.3 & 0 & 0.0 \\
Alpacas tuis & 140 & 140 & 100.0 & 53 & 37.9 \\
Alpacas adultas & 15 & 15 & 100.0 & 10 & 66.7 \\
Llama & 15 & 14 & 93.3 & 9 & 60.0 \\
Conejo Angora & 15 & 15 & 100.0 & 5 & 33.3 \\
Paco-vicuña & 15 & 15 & 100.0 & 8 & 53.3 \\
\hline
\end{tabular}


Cuadro 2. Porcentaje de medulación y estadísticos relacionados de fibras de lana de ovino, alpacas, llamas, conejos y paco-vicuña

\begin{tabular}{lcccccc}
\hline Especie & $\mathrm{n}$ & $\begin{array}{c}\text { Promedio } \\
(\%)\end{array}$ & $\begin{array}{c}\text { Desviación } \\
\text { estándar } \\
(\%)\end{array}$ & $\begin{array}{c}\text { Valor } \\
\text { mínimo } \\
(\%)\end{array}$ & $\begin{array}{c}\text { Valor } \\
\text { máximo } \\
(\%)\end{array}$ & $\begin{array}{c}\text { Error } \\
\text { estándar } \\
(\%)\end{array}$ \\
\hline Ovino & 15 & 0.35 & 0.62 & 0 & 1.92 & 0.16 \\
Alpacas tuis & 140 & 22.61 & 17.52 & 2.83 & 80.43 & 1.48 \\
Alpacas adultas & 15 & 35.55 & 18.81 & 11.90 & 66.33 & 4.86 \\
Llama & 15 & 39.96 & 26.74 & 0 & 85.43 & 6.91 \\
Conejo & 15 & 92.2 & 9.66 & 66.83 & 100.00 & 2.49 \\
Paco vicuña & 15 & 17.04 & 9.79 & 2.94 & 34.74 & 2.53 \\
\hline
\end{tabular}

Cuadro 3. Porcentaje de medulación y media de diámetro de fibra (MDF) \pm error estándar según tipo de medulación de lana y fibras de diferentes especies animales

\begin{tabular}{|c|c|c|c|c|c|}
\hline Tipo de medulación & $\begin{array}{l}\text { Ovino } \\
(\mathrm{n}=15)\end{array}$ & $\begin{array}{c}\text { Alpacas } \\
\text { adultas } \\
(\mathrm{n}=15)\end{array}$ & $\begin{array}{l}\text { Conejo } \\
(\mathrm{n}=15)\end{array}$ & $\begin{array}{l}\text { Llama } \\
(\mathrm{n}=15)\end{array}$ & $\begin{array}{l}\text { Paco-vicuña } \\
\quad(\mathrm{n}=15)\end{array}$ \\
\hline \multicolumn{6}{|c|}{ No meduladas } \\
\hline Porcentaje (\%) & $99.65 \pm 0.16$ & $64.45 \pm 4.86$ & $7.80 \pm 2.49$ & $60.04 \pm 6.91$ & $82.96 \pm 2.53$ \\
\hline $\operatorname{MDF}(\mu \mathrm{m})$ & $19.78 \pm 0.63$ & $19.71 \pm 0.48$ & $14.72 \pm 0.67$ & $23.47 \pm 1.23$ & $12.58 \pm 0.31$ \\
\hline \multicolumn{6}{|c|}{ Meduladas } \\
\hline \multicolumn{6}{|l|}{ Fragmentadas } \\
\hline Medulación (\%) & $0.32 \pm 0.15$ & $16.62 \pm 2.18$ & $8.67 \pm 1.49$ & $13.35 \pm 2.47$ & $7.69 \pm 1.42$ \\
\hline $\operatorname{MDF}(\mu \mathrm{m})$ & $15.40 \pm 0.86$ & $23.57 \pm 0.56$ & $14.38 \pm 0.59$ & $24.98 \pm 0.68$ & $15.13 \pm 0.43$ \\
\hline \multicolumn{6}{|l|}{ Discontinuas } \\
\hline Medulación (\%) & 0 & $13.11 \pm 2.01$ & $31.42 \pm 2.65$ & $17.03 \pm 3.3$ & $5.15 \pm 0.98$ \\
\hline $\operatorname{MDF}(\mu \mathrm{m})$ & - & $26.41 \pm 0.48$ & $15.36 \pm 0.43$ & $28.99 \pm 0.76$ & $17.22 \pm 0.49$ \\
\hline \multicolumn{6}{|l|}{ Continuas } \\
\hline Medulación (\%) & $0.03 \pm 0.03$ & $3.73 \pm 0.7$ & $51.15 \pm 4.15$ & $8.2 \pm 2.03$ & $3.5 \pm 0.74$ \\
\hline $\operatorname{MDF}(\mu \mathrm{m})$ & 12.47 & $26.45 \pm 2.04$ & $16.2 \pm 0.38$ & $30.71 \pm 0.85$ & $20.1 \pm 1.12$ \\
\hline \multicolumn{6}{|l|}{ Tipo kemp } \\
\hline Medulación (\%) & 0 & $2.10 \pm 0.69$ & $0.96 \pm 0.62$ & $1.38 \pm 0.56$ & $0.7 \pm 0.22$ \\
\hline $\operatorname{MDF}(\mu \mathrm{m})$ & - & $39.46 \pm 2.31$ & $33.09 \pm 5.58$ & $56.18 \pm 9.91$ & $46.05 \pm 3.24$ \\
\hline
\end{tabular}


nen una baja tasa de medulación (17.04\%). Asimismo, presentan los cuatro tipos de medulación (fragmentada, discontinua, continua y kemp). La fibra de conejo, aunque tuvo una alta tasa de medulación (92.2\%) tuvo una media de diámetro bajo (14.72 \pm 0.67$)$, indicando que las fibras finas también tienen médula en su estructura.

La lana de ovino y las fibras de alpaca presentaron medias de diámetro similares (alrededor de $19.7 \mu \mathrm{m}$ ), aunque la lana está conformada principalmente por fibras sin médula, mientras que las fibras de alpaca tienen una incidencia de medulación por encima del 35\%. Por otro lado, las fibras de alpaca y llama tienen similares porcentajes de medulación; sin embargo, las fibras de alpaca son más finas que de la llama, pero ambas son más gruesas que las fibras de paco vicuña y de conejo Angora.

Es de notar un patrón característico, en torno a la media de diámetro y el porcentaje de medulación de las fibras, pues a excepción de la lana de ovino, el MDF incrementa en forma progresiva en casi todas las demás especies, desde las fibras con medulación fragmentada, pasando a la discontinua, continua y terminando con las fuertemente meduladas. Del mismo modo, las fibras no meduladas resultan ser más finas que las fibras con cualquier tipo de medulación (Cuadro 3, Figura 2).

Los porcentajes de medulación de fibras de alpacas tuis machos y alpacas adultas fueron de $22.61 \pm 1.48$ y $35.65 \pm 4.86 \%$, respectivamente $(\mathrm{p}<0.05)$. Asimismo, el mayor porcentaje por tipo de medulación se observó en las fibras con medulación fragmentada, seguida de la medulación continua, discontinua y fuertemente medulada $(9.17 \pm 0.66$, $7.85 \pm 0.68,4.86 \pm 0.43$ y $0.72 \pm 0.14 \%$, respectivamente); sin embargo, son más numerosas las fibras sin medulación en comparación a las meduladas (Cuadro 4).

\section{Relación entre Medulación y Diámetro de Fibra}

En los tipos de fibras analizados, se observa una aparente relación directa entre la incidencia de medulación con la MDF (eva-
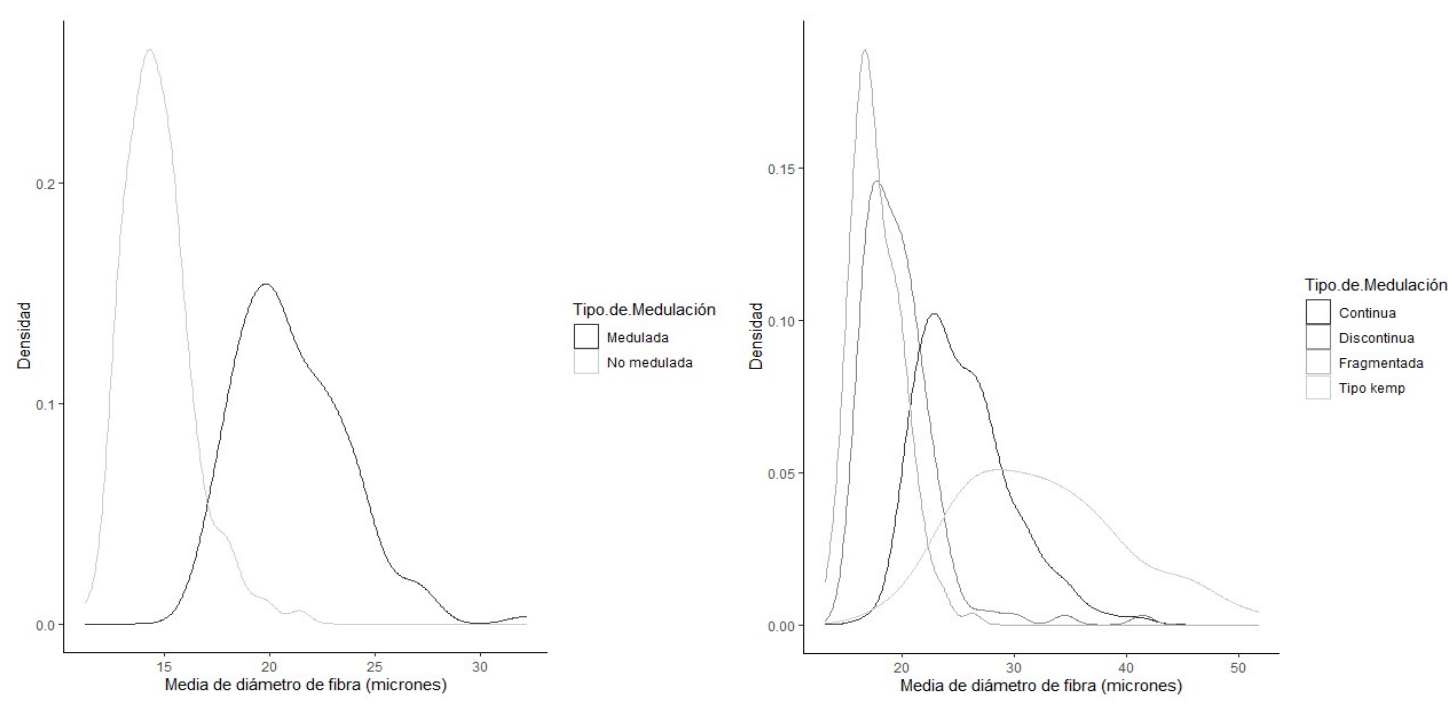

Figura 2. Diagramas de densidad del diámetro de fibras por tipo de medulación. Izquierda: Medias de diámetro de fibras meduladas y no meduladas. Derecha: Medias de diámetro de fibras por tipo de medulación 
Cuadro 4. Estadísticos descriptivos del porcentaje de medulación, según tipo de fibras meduladas de alpacas tuis Huacaya de color blanco

\begin{tabular}{lcccccc}
\hline Tipo de medulación & $\mathrm{n}$ & Promedio & $\begin{array}{c}\text { Desviación } \\
\text { estándar }\end{array}$ & $\begin{array}{c}\text { Valor } \\
\text { mínimo }\end{array}$ & $\begin{array}{c}\text { Valor } \\
\text { máximo }\end{array}$ & $\begin{array}{c}\text { Error } \\
\text { estándar }\end{array}$ \\
\hline No meduladas (\%) & 140 & 77.39 & 17.52 & 19.57 & 97.17 & 1.48 \\
Meduladas (\%) & 140 & 22.61 & 17.52 & 2.83 & 80.43 & 1.48 \\
$\quad$ Fragmentadas (\%) & 140 & 9.17 & 7.79 & 0.67 & 45.00 & 0.66 \\
Discontinuas (\%) & 140 & 4.86 & 5.09 & 0.00 & 29.17 & 0.43 \\
Continuas (\%) & 140 & 7.85 & 8.09 & 0.50 & 58.04 & 0.68 \\
Tipo kemp (\%) & 140 & 0.72 & 1.68 & 0.00 & 9.82 & 0.14 \\
\hline
\end{tabular}

Cuadro 5. Coeficiente de correlación de Pearson (r), intervalo de confianza (IC) de $\mathrm{r}$ y pvalor, de la media del diámetro de fibra (MDF) con logaritmo de medulación total y medulación tipo kemp de fibras de cinco especies animales

\begin{tabular}{lccccccc}
\hline \multirow{2}{*}{$\begin{array}{l}\text { Especie } \\
\text { gl }\end{array}$} & \multicolumn{4}{c}{$\begin{array}{c}\text { MDF con Log de Medulación } \\
\text { total }\end{array}$} & \multicolumn{3}{c}{$\begin{array}{c}\text { MDF con Log de Medulación } \\
\text { tipo kemp }\end{array}$} \\
\cline { 2 - 8 } & & $\mathrm{r}$ & $\mathrm{IC}(95 \%)$ & $\mathrm{p}$-valor & $\mathrm{r}$ & $\mathrm{IC}(95 \%)$ & $\mathrm{p}$-valor \\
\hline Ovino & 13 & 0.17 & {$[-0.38+0.63]$} & 0.550 & - & - & - \\
Alpacas tuis & 138 & 0.46 & {$[+0.32+0.58]$} & 0.001 & 0.41 & {$[+0.26+0.54]$} & 0.001 \\
Alpacas adultas & 13 & 0.65 & {$[+0.21+0.87]$} & 0.008 & 0.45 & {$[-0.08+0.78]$} & 0.091 \\
Conejos Angora & 9 & 0.23 & {$[-0.43+0.72]$} & 0.502 & 0.43 & {$[-0.23+0.82]$} & 0.189 \\
Llama & 13 & 0.41 & {$[-0.12+0.76]$} & 0.124 & 0.28 & {$[-0.28+0.69]$} & 0.320 \\
Paco-vicuña & 13 & 0.57 & {$[+0.09+0.83]$} & 0.026 & 0.04 & {$[-0.48+0.54]$} & 0.875 \\
\hline
\end{tabular}

gl: Grados de libertad

Cuadro 6. Estadísticos de regresión lineal simple de la media de diámetro de fibra (MDF, variable dependiente) sobre el porcentaje de medulación total transformado (variable independiente) en fibras de cinco especies animales

\begin{tabular}{lccccc}
\hline \multirow{2}{*}{ Especie } & & \multicolumn{4}{c}{ Regresión de la MDF sobre el porcentaje de medulación } \\
& $\mathrm{gl}$ & \multicolumn{4}{c}{ total transformado } \\
\cline { 3 - 6 } & & $\mathrm{a}$ & $\mathrm{b}_{\mathrm{MDF}, \% \mathrm{Med}} \pm \mathrm{EE}$ & $\mathrm{p}$-valor & $\mathrm{R}^{2}$ \\
\hline Ovino & 13 & 19.75 & - & - & - \\
Alpacas tuis & 138 & 19.95 & $1.11 \pm 0.20$ & 0.001 & 0.19 \\
Alpacas adultas & 13 & 21.87 & $0.72 \pm 0.40$ & 0.091 & 0.20 \\
Conejo Angora & 9 & 15.54 & $0.41 \pm 0.29$ & 0.189 & -0.18 \\
Llama & 13 & 26.08 & $1.03 \pm 0.99$ & 0.320 & 0.08 \\
Paco-vicuña & 13 & 13.43 & $0.05 \pm 0.32$ & 0.875 & 0.01 \\
\hline
\end{tabular}

gl: Grados libertad; a: Intercepto; $b_{M D F, \% M e d}$ : Coeficiente de regresión; $\mathrm{R}^{2}$ : Coeficiente de determinación 


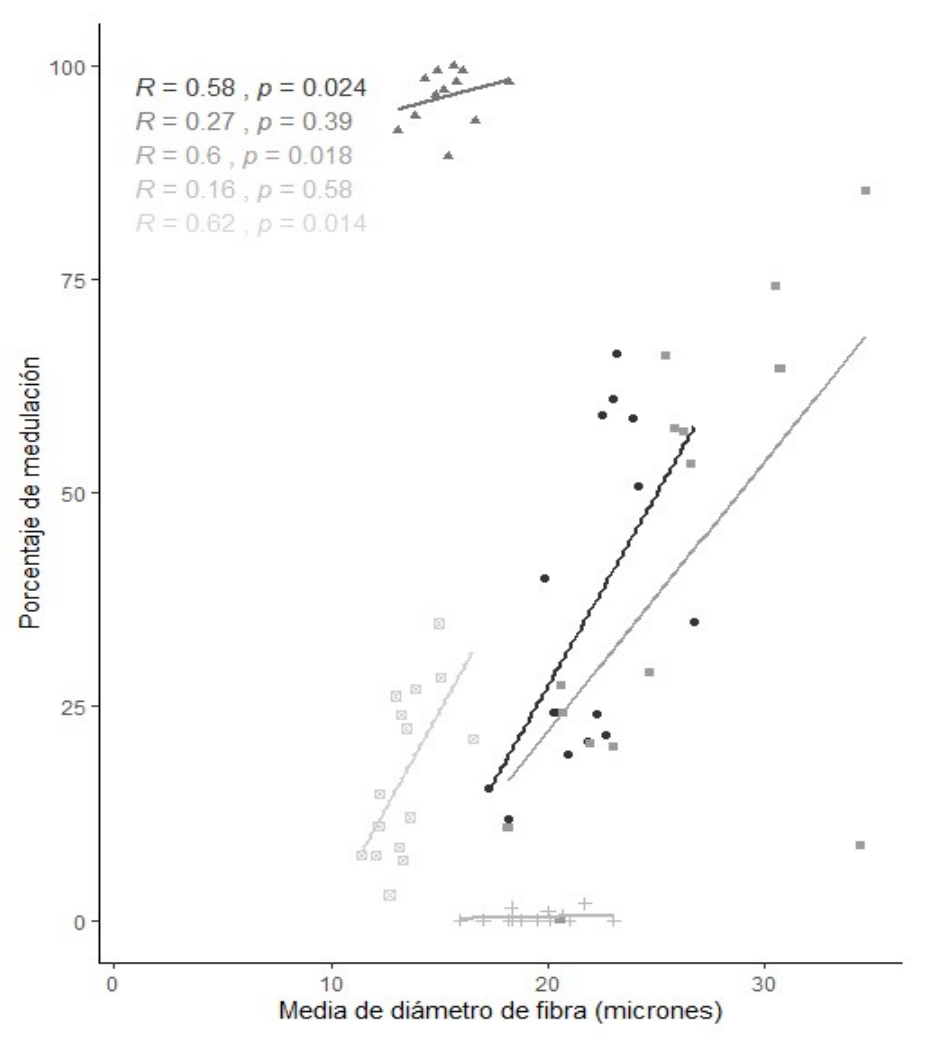

ESPECIE

a Alpaca

ta Angora

- Llama

वे Ovino

-E. Paco Vicuña

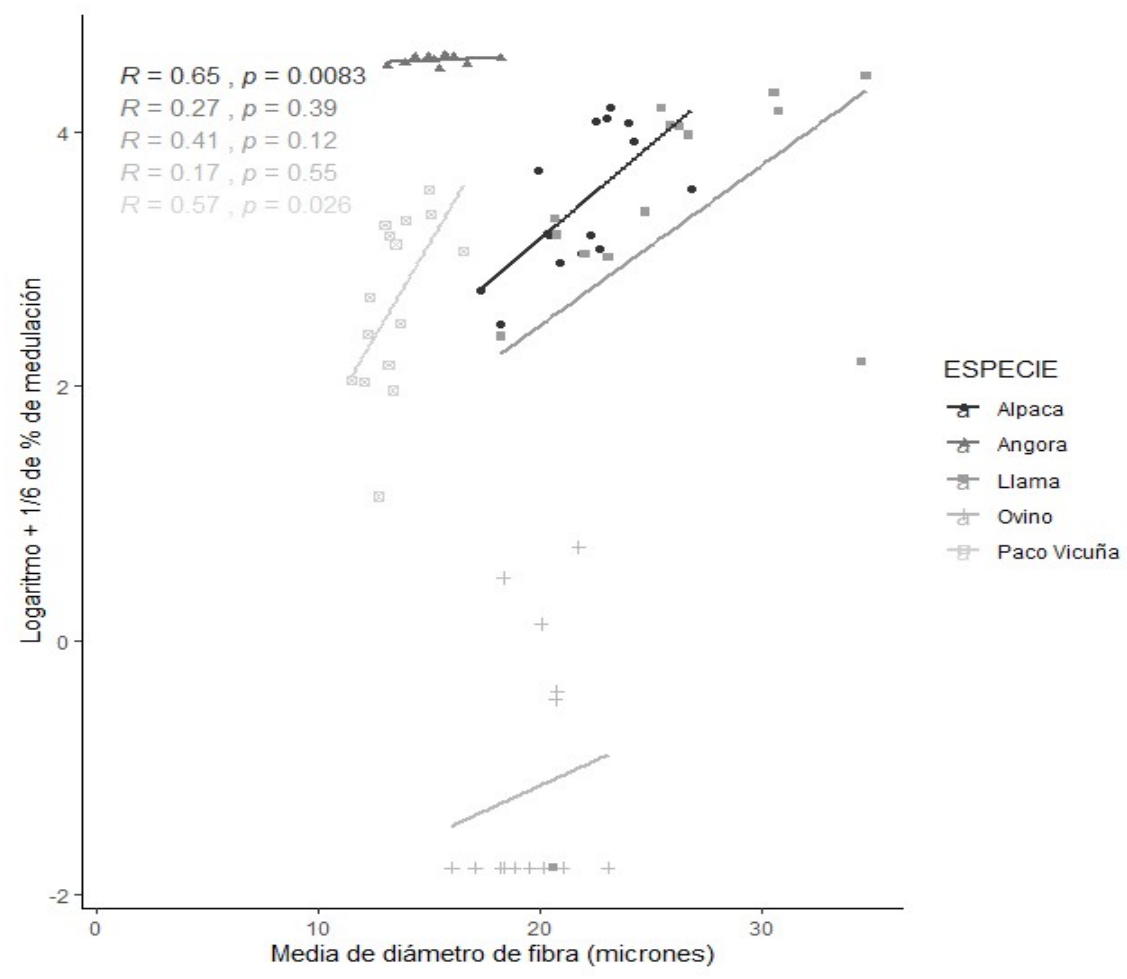

Figura 3. Diagramas de dispersión con ajuste de línea de regresión de la media del diámetro de fibra (MDF) y el porcentaje de medulación. Arriba: Sin transformación; Abajo: Con transformación logarítmica más 1/6) de fibras de alpaca, conejo Angora, llama, de lana de ovino y paco vicuña. Se incluye los coeficientes de correlación de Pearson 
Cuadro 7. Estadísticos de regresión lineal simple de la media de diámetro de fibra (MDF) con el porcentaje transformado de medulación en fibras de alpacas Huacaya tuis de color blanco, según tipo de medulación

\begin{tabular}{lccccc}
\hline \multirow{2}{*}{$\begin{array}{c}\text { Regresión de MDF con el } \\
\text { porcentaje transformado de }\end{array}$} & gl & \multicolumn{4}{c}{ Estadísticos de la regresión } \\
\cline { 3 - 6 } medulación & 138 & 19.95 & $1.11 \pm 0.20$ & 0.001 & 0.19 \\
\hline Total & 138 & 17.55 & $0.81 \pm 0.19$ & 0.001 & 0.12 \\
Fragmentada & 138 & 18.42 & $0.59 \pm 0.15$ & 0.001 & 0.10 \\
Discontinua & 138 & 15.54 & $0.41 \pm 0.16$ & 0.001 & 0.10 \\
Continua & 138 & 19.69 & $0.63 \pm 0.14$ & 0.001 & 0.13 \\
Tipo kemp & & & $\mathrm{b} \pm \mathrm{EE}$ & $\mathrm{p}$-valor & $\mathrm{R}^{2}$ \\
\hline
\end{tabular}

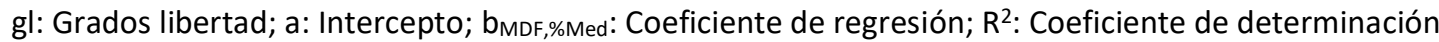

luada mediante el coeficiente de correlación de Pearson: r). En el análisis estadístico en fibras de alpacas tuis, alpacas adultas y de paco-vicuña se encontró correlaciones positivas entre moderada y alta entre dichas variables $(0.65,0.46$ y 0.57 , respectivamente). También se encontró que en fibras de alpacas tuis existe una moderada correlación entre la incidencia de medulación tipo kemp con la MDF (Figura 3, Cuadro 5). Por otro lado, en lana de ovinos Merino se encontró correlaciones bajas no significativas, probablemente debido las bajas o nulas incidencias de medulación.

Los coeficientes de correlación en fibras de alpacas y paco-vicuñas indican que a mayor porcentaje de medulación corresponde mayor diámetro de las fibras. En el análisis de regresión, el coeficiente de regresión de la MDF sobre el logaritmo del porcentaje de medulación resulta significativo a nivel del $95 \%\left(b_{\text {MDF,Log Med })}=1.11 \pm 0.2\right)$ solo en alpacas tuis, en tanto que en alpacas adultas resulta significativo a un nivel del $90 \%$. Los coeficientes de regresión no fueron significativos para las fibras de las demás especies (Figura 3, Cuadro 6).
En fibras de alpacas tuis también se encontró una relación directa entre los porcentajes transformados de los diferentes tipos de medulación (porcentaje de medulación total, fibras fragmentadas, discontinuas, continuas y tipo kemp) con la MDF (Cuadro 7), con coeficientes de regresión que varían entre 0.41 y 1.1 y con coeficientes de determinación moderados $\left(\mathrm{r}^{2}\right.$ entre 0.10 y 0.19$)$, que permite afirmar que a mayor incidencia de cualquier tipo de medulación corresponde una mayor MDF (Figura 3). Bajo estas consideraciones, con fines predictivos, se puede indicar que por cada unidad de incremento del porcentaje de medulación total transformado, la MDF incrementa en $1.11 \pm 0.20 \mu \mathrm{m}$, siendo menor el incremento cuando se aumenta en una unidad los otros porcentajes de tipo de medulación transformados (Cuadro 7).

En una vista transversal, las fibras de lana son generalmente redondeadas, observándose ocasionalmente alguna medulación; sin embargo, las fibras de alpaca, llama y paco-vicuña varían de forma redonda a ovalada, observándose que las fibras más gruesas y con mayor diámetro de médula tienden a ser mayormente ovaladas. De otro lado, 

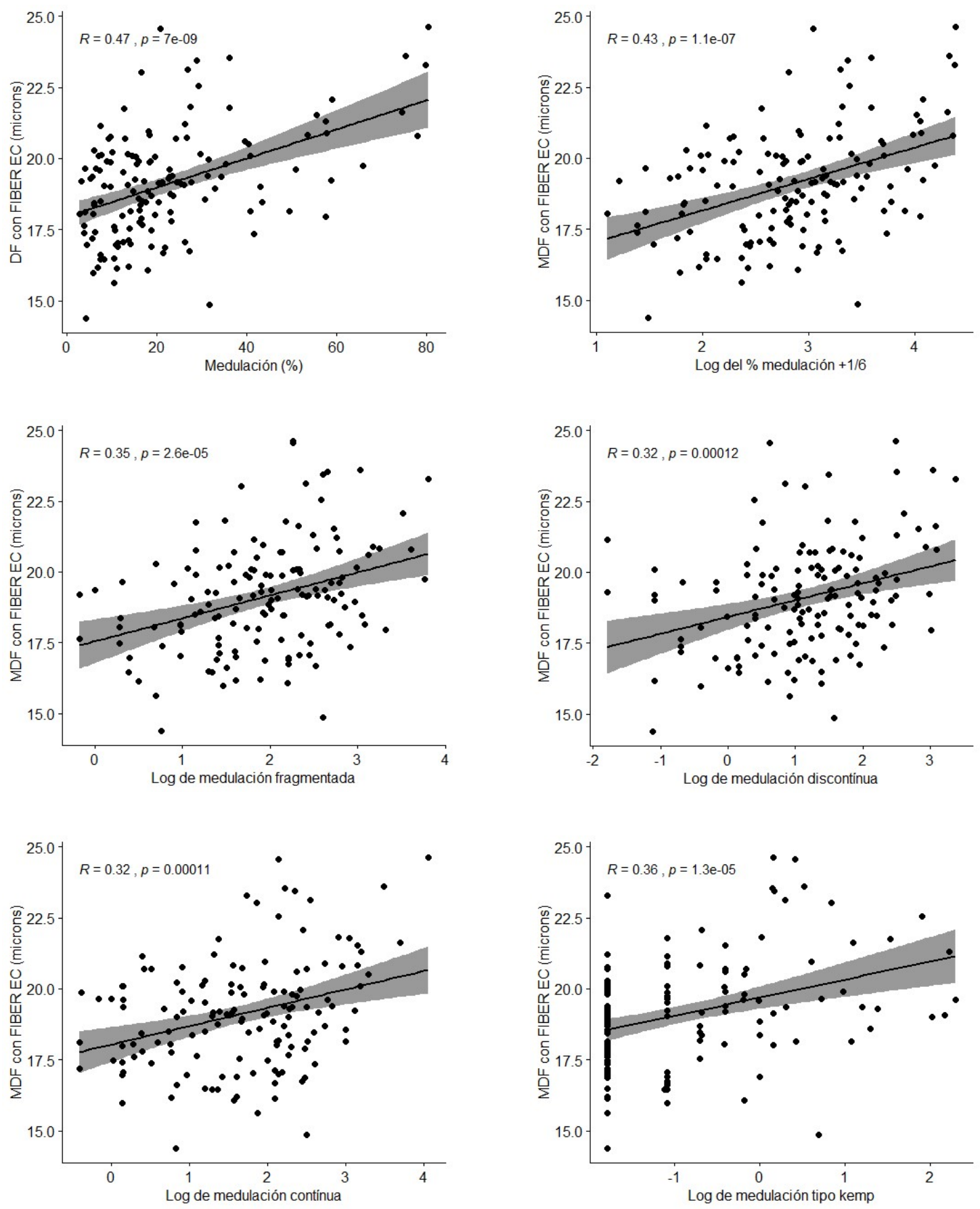

Figura 4. Diagramas de dispersión con ajuste de línea de regresión de la media de diámetro de fibra (MDF) y el logaritmo del porcentaje de medulación según tipos de medulación en fibras de alpacas tuis Huacaya de color blanco 

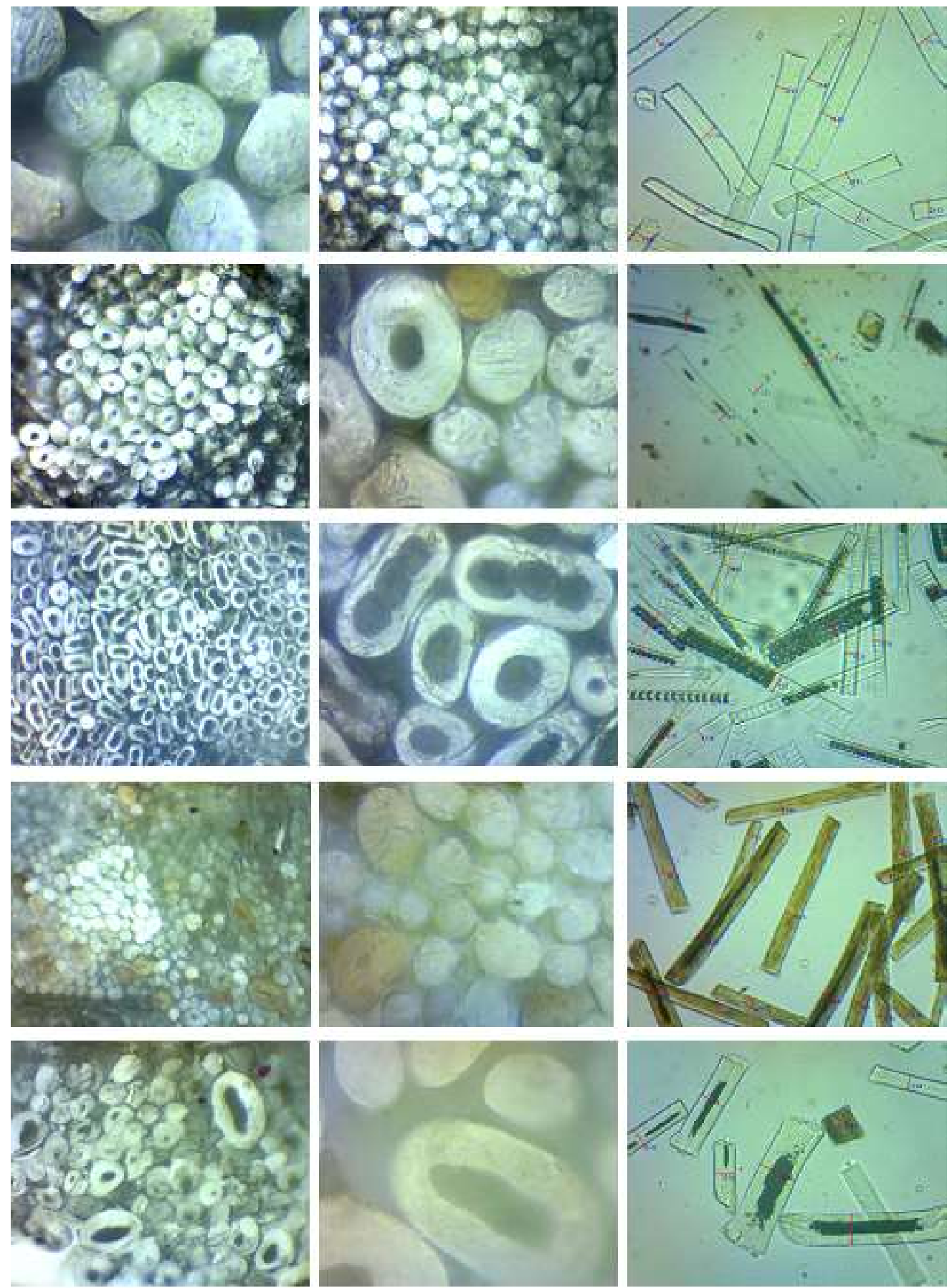

Figura 5. Vistas fotográficas de cortes transversales y longitudinales de lanas y fibras de diferentes especies de animales. De arriba hacia abajo: lana de ovino, fibras de alpaca, conejo angora, paco vicuña y llama 
las fibras de conejo Angora son generalmente todas meduladas, pocas de forma redondeada, predominando médulas de forma lobulada, elíptica, bastante ovaladas y en algunas casi aplanadas. Estas se logran ver en fibras más gruesas, encontrándose entre dos $\mathrm{y}$ tres canales medulares, mientras que la presencia de medulas casi tubulares o levemente ovales se visualizan en las fibras finas y redondeadas. Las fibras de conejo vistas longitudinalmente tienen forma reticular o escalera, siendo esta de tipo uni o multiserial; es decir, apareciendo como si hubiera varias escaleras una al lado de otra (Figuras 4 y 5).

\section{Discusión}

La incidencia, porcentaje y forma de medulación resultan ser características propias de fibras de cada especie animal (McGregor, 2012). En estado silvestre, los animales cubiertos por fibras, tienen doble capa de fibras (Vásquez et al., 2000), unas son fibras finas («down») y otras muy gruesas («pelos o cerdas»), las que tienen alta incidencia de medulación (Antonini et al., 2004; Allain y Renieri, 2010); sin embargo, al pasar por el proceso de domesticación y debido a la aplicación de técnicas de mejoramiento animal en animales que producen lana y fibras, se optó por disminuir el diámetro de fibra, lo cual conllevó también a disminuir la incidencia de medulación. Estas explicaciones sustentarían que, en ovinos Merino, son pocos los animales cuyo vellón contiene vestigios de fibras meduladas. Los camélidos domésticos también fueron sometidos a procesos de mejoramiento genético mediante selección, habiéndose logrado disminuir el grosor en alpacas más no la incidencia de fibras meduladas, probablemente debido que las técnicas de selección empleadas no han sido del todo bien encaminadas y eficientes (Pinares et al., 2018). Contrariamente, las fibras de conejo Angora son generalmente meduladas, pero a la vez finas (Chattopadhyay et al., 2005), razón por la cual tienen una baja densidad y al confeccionar prendas en base a ella, resultan ser muy livianas (Süpüren et al., 2014).

La existencia de medulación en todas las muestras de camélidos (caso excepcional de una muestra de llama donde no se encontró medulación alguna), así como de medulación tipo «kemp» (en más del 33\%), se debería a factores intrínsecos (genético, especie y variedad animal). Sin embargo, algunos factores extrínsecos pueden tener efecto sobre la medulación, pues se sabe que las alpacas, llamas y paco-vicuñas son criadas generalmente en condiciones extensivas y bajo sistemas de bajos recursos logísticos y de infraestructura (Quispe et al., 2009) y, por tanto, propensos a sufrir el efecto de la alimentación, manejo y sanidad, efectos que han sido discutidos y explicados por McGregor (2012) y Radzik et al. (2018); en tanto que efectos de edad, color y ubicación dentro de la zona corporal han sido comentados por Martínez et al. (1997).

El bajo porcentaje de medulación en las fibras de lana de ovinos Merino concuerda con los reportes de Ferguson et al. (2012) y McGregor (2012), quienes afirman que la medulación se presenta en forma esporádica. De otro lado, los porcentajes de medulación encontrados en fibras de alpacas adultas y alpacas tuis de color blanco son ligeramente mayores o similares, respectivamente, a los reportados por Lupton et al. (2006) y Cruz et al. (2019) y dentro del rango presentado por McGregor (2006). No obstante, otros autores reportan valores inferiores (Contreras, 2010; Ferguson et al., 2012; Córdova, 2015; Radzik et al., 2018; Pinares et al., 2019). Estas diferencias podrían deberse al método de medición empleado (Ferguson et al. [2012] utilizaron el OFDA 100), el lugar de crianza (Radzik et al. [2018] trabajaron con alpacas en Australia, África y Europa) y al rango de diámetro de fibras (Córdova [2015] trabajó con fibras de 25-29 $\mu \mathrm{m}$ y en este estudio fue de $19.11 \pm 0.16 \mu \mathrm{m}$ y $21.81 \pm 0.63 \mu \mathrm{m}$ en alpacas tuis y adultas, respectivamente). 
La mayor tasa de medulación de las fibras de camélidos, comparados con fibras de lana, generaría una menor densidad a las fibras de alpacas y llamas (Czaplicki, 2012). Asimismo, podría contribuir a explicar el efecto aislante, dado que la fibra de alpaca tiene mayor capacidad aislante que la lana, lo cual se debería a los espacios vacíos existentes dentro de la fibra (médula) que atraparían el aire del medioambiente (Wang et al., 2005). Por tanto, la medulación en fibras de camélidos más que un defecto podría ser una bondad (McGregor, 2012). Las fibras de medulación fragmentada, discontinua y continua no tienen un grosor exagerado (MDF menor a $26.5 \mu \mathrm{m}$ ); sin embargo, las fibras que tienen medulación tipo «kemp», que son las llamadas fibras objetables tienen un serio defecto (ASTM, 1986; Lupton y Pfeiffer, 1998), pues producen el efecto picazón en alpacas (McGregor, 2006) y en llamas (Cochi, 1999; Frank et al., 2009).

Las fibras tipo kemp encontradas en alpacas adultas y llamas (MDF 39.5 \pm 2.3 y $56.2 \pm 9.9 \mu \mathrm{m}$, respectivamente) serían las que producen la sensación incómoda de picazón. Sin embargo, gran parte de este tipo de fibras pueden ser eliminados mediante el descerdado (Cochi, 1999; Wang et al., 2003; Frank et al., 2009; Poma, 2018). La selección animal sería otro proceso que permitiría disminuir la tasa de medulación tipo «kemp», pues esta característica tiene una moderada heredabilidad (Pinares et al., 2018), que permitiría tener un rápido avance genético.

El $92.2 \pm 2.5 \%$ de medulación encontrado en fibras de conejo Angora se ubica dentro del rango reportado (Herrmann et al., 1996; Blacutt, 2002; Onal et al., 2007; Rafat et al., 2007), aunque inferior al hallazgo de Chattopadhyay et al. (2005) en conejos Angora de la India, habiéndose demostrado que el origen y el lugar de crianza tienen efecto sobre la tasa de medulación (Herrmann et al., 1996).
En base a las correlaciones encontradas, la deducción de la relación directa entre porcentaje de medulación y la MDF concuerda con reportes en alpacas (Moore, 2015; McGregor, 2006). Asimismo, Radzik et al. (2018) encontraron que a medida que aumenta el grosor de las fibras disminuye el porcentaje de fibras no meduladas y de medulación fragmentada, pero incrementa la medulación continua. En llamas, Martínez et al. (1997) concluyeron que el porcentaje de medulación se incrementa con la edad y el mayor diámetro de fibra de llama, en tanto que Onal et al. (2007) encontraron correlaciones positivas mayores a 0.88 con la MDF.

La existencia de medulación en fibras finas es respaldada por Radzik et al. (2018), quienes, trabajando en alpacas, encontraron médulas en fibras con diámetros entre $14 \mathrm{y}$ $20 \mu \mathrm{m}$. Similarmente, Radzik et al. (1995) encontraron fibras meduladas en lanas finas de ovinos nativos de la India, aunque McGregor (2006) indica que en fibras de alpacas con MDF de $18 \mu \mathrm{m}$ a menos no se encontraría fibras meduladas.

Para fines de mejoramiento de la calidad de las fibras de alpacas y llamas (a través de la selección animal) resulta conveniente considerar la relación directa entre el diámetro (MDF) y el porcentaje de medulación $(\% \mathrm{M})$, pues al seleccionar alpacas de menor MDF indirectamente se obtendrían animales con menores porcentajes de medulación, o viceversa (Gupta et al., 1981, Cruz et al., 2019). Ala fecha, el porcentaje de medulación no es considerado como criterio de selección, lo cual se debería a tres razones. Primero, a la fecha no existe un equipo que mida con buena precisión, exactitud y en forma práctica la medulación de la fibra de alpacas y llamas; segundo, porque no todas las fibras meduladas tienen un efecto negativo sobre la calidad de las fibras y por tanto no sería recomendable disminuir el porcentaje global de medulación; y tercero, porque solamente las fibras con medulación continua y especial- 
mente las fibras tipo kemp o fibras objetables deberían ser eliminadas o drásticamente disminuidas a fin de mejorar la calidad y, por tanto, el precio de las fibras (Frank et al., 2009).

Así, aunque las alpacas producen vellones con fibras finas, (Quispe et al., 2009; Omachea et al., 2015; Vásquez et al., 2015, Pinares et al., 2018) - los cuales tendrían bajos porcentajes de medulación -, aún se tiene el problema del picazón, razón por la cual, algunos investigadores como Pinares et al. (2018) y Cruz et al. (2019), entre otros, vienen proponiendo considerar al porcentaje de fibras con medulación continua y tipo kemp como criterio de selección para mejorar la calidad de la fibra, pues a la fecha la disminución de la MDF pasaría ser relegada (Pinares et al., 2018).

Las vistas transversales de las fibras de lana Merino redondeadas estarían relacionadas con una MDF baja. Así, Frank et al. (2009) afirman que las fibras finas, al corte transversal, normalmente tienen una forma casi circular o apenas elíptica. McGregor (2012), asimismo, afirma que este patrón también se observa en fibras especiales. Los hallazgos del presente estudio en torno a las diferentes formas y tamaños de los canales medulares de las fibras de llamas y alpacas, concuerda con otros reportes que indican que la forma transversal de las médulas varía de acuerdo al rango de la MDF, de modo que las fibras que tienen médulas son redondeadas, mientras que en las fibras medianas y gruesas aparecen formas más irregulares (Frank et al., 2009; Allain y Renieri, 2010; Czaplicki, 2012).

Las vistas transversales de fibras de conejo, registradas en el presente trabajo, concuerdan con las reportadas por Chattopadhyay et al. (2005) y Allain y Renieri (2010), quienes encontraron fibras con uno a tres canales medulares. Asimismo, las vistas longitudinales observadas en este trabajo son similares a las medulaciones de tipo reticular encontrados por Langley y Kennedy (1981) y Onal et al. (2007), mientras que Süpüren et al. (2014) reportaron medulaciones tipo escalera uni y multiserial, que van de formas ovales a rectangulares.

Bajo estas consideraciones se puede indicar que en fibras de alpacas con mayor diámetro al corte transversal se encuentran fibras de forma de elíptica, con similares características en sus médulas. Esto no puede ampliarse a las fibras de conejos que, a pesar de ser bastante finas, las fibras en casi su totalidad son muy elípticas y con médulas lobuladas, principalmente en fibras de forma ovoide alargada. McGregor y Quispe (2018) encontraron que la elipticidad se incrementa en 0.006 por cada micrómetro en la MDF.

\section{Conclusiones}

- La tasa de medulación en ovinos Merinos es cercana a cero, en conejos Angora es cercana al $100 \%$ y en fibras de camélidos varía entre 17 y $40 \%$, observándose menores tasas de medulación en paco-vicuña y alpacas tuis machos comparados con alpacas adultas.

- Existe una relación directa entre la medulación con el diámetro de las fibras; sin embargo, la presencia de fibras meduladas se observa tanto en fibras gruesas como en fibras finas.

- La forma de las fibras y las médulas varían según las especies; sin embargo, las fibras finas, tanto las fibras como las médulas son redondeadas, en tanto que en fibras gruesas ambas son bastante elípticas.

\section{Literatura Citada}

1. Allain D, Renieri C. 2010. Genetics of fibre production and fleece characteristics in small ruminants, Angora rabbit and South American camelids. Animal 4: 1472-1481. doi: 10.1017/S1751731110000029 . 
2. Antonini M, Gonzales M, Valbonesi A. 2004. Relationship between age and postnatal skin follicular development in three types of South American domestic camelids. Liv Produc Sci 90: 241-246.

3. [ASTM] American Society for Testing and Materials. 1986. Standard test method for medullation and kemp fibers in wool and other animal fibers by microprojection, D2968, Annual Book of ASTM Standards. Philadelphia, Pennsylvania.

4. Balasingam A. 2005. The definitions of medullation threshold values used by different testing methods to define an objectionable medullated fibre in Merino Wool. Australian Wool Testing Authority Ltda. $32 \mathrm{p}$.

5. Blacutt JJ. 2002. Rendimiento y calidad de fibra de conejos Angora en diferentes esquilas. Tesis de Grado. La Paz, Bolivia: Univ. Mayor de San Andrés. 58 p.

6. Cochi N. 1999. Determinación del rendimiento y calidad de la fibra descerdado de llamas (Lama glama). Tesis de Grado. La Paz, Bolivia: Univ. Mayor de San Andrés. Bolivia. $120 \mathrm{p}$.

7. Contreras A. 2010. Estructura y características físicas de la fibra de alpaca Huacaya (Vicugna pacos) de color blanco. Tesis de Ingeniero Zootecnista. Huancavelica, Perú: Univ. Nacional de Huancavelica.

8. Córdova ML. 2015. Comparación de la calidad de las fibras de Vicugna pacos (alpaca) y Lama lama (llama). Tesis de Ingeniero Zootecnista. Riobamba, Ecuador: Escuela Superior Politécnica de Chimborazo. $113 \mathrm{p}$.

9. Cruz A, Morante R, Gutiérrez JP, Torres A, Burgos A, Cervantes I. 2019. Genetic parameters for medullated fiber and its relationship with other productive traits in alpacas. Animal 13: 1358-1364. doi: $10.1017 / \mathrm{S} 1751731118003282$

10. Czaplicki Z. 2012. Properties and structure of polish alpaca wool. Fibres Text East Eur 20: 8-12.
11. Chattopadhyay SK, Bhaskar P, Ahmed M, Gupta NP, Pokharna AK. 2005. Properties of indigenous angora rabbit fair and cotton blended yarns spun using short-staple cotton spinning system. Indian J Fibre Text 30: 215-217.

12. Ferguson MB, McGregor $B A$, Behrendt R. 2012. Relationships between skin follicle characteristics and fibre properties of Suri and Huacaya alpacas and Peppin Merino sheep. Anim Prod Sci 52: 442-447. doi: 10.1071/ AN11233

13. Frank EA, Hick MVH, Adot OG. 2009. Descriptive differential attributes of type of fleeces in llama fibre and its textile consequence. Part 2: consequences of the dehairing process. J Text I 102: 41-49. doi: 10.1080/00405000903474873

14. Gupta NP, Arora RK, Verma GK. 1981. An assessment of the characteristics of medullated and nonmedullated wool fibres. Indian J Text Res 6: 92-95.

15. Herrmann S, Wortmann G, Wortmann FJ. 1996. Characteristics of Angora rabbit Fibre 1 - The influence of fibre origin on fibre and medulla diameter in Angora wool. World Rabbit Sci 4: 149153. doi: $10.4995 /$ wrs. 1996.287

16. Hunter L. 1993. Mohair: a review of its properties, processing and applications. CSIRO Division of Textile Technology. Port Elizabeth, South Africa: NMB Printers (Pty). 278 p.

17. [INDECOPI] Instituto Nacional de Defensa de la Competencia y de la Protección de la Propiedad Intelectual. 2014. Fibra de alpaca clasificada. Definiciones, clasificación por grupos de calidades, requisitos y rotulado. Norma Técnica Peruana. Comisión de Normalización y de Fiscalización de Barreras Comerciales no Arancelarias, Lima, Perú.

18. Lupton CJ, McColl A, Stobart RH. 2006. Fiber characteristics of the Huacaya alpaca. Small Rumin Res 64: 
211-224. doi: 10.1016/j.smallrumres.2005.04.023

19. Langley K, Kennedy T. 1981. The identification of specialty fibers. Text Res J 51: 703-709. doi: 10.1177/004051758105101104

20. Lupton CJ, Pfieffer FA. 1998. Measurement of medullation in wool and mohair using an optical fibre diameter analyser. J Anim Sci 76: 1261-1266. doi: 10.2527/1998.7651261x

21. Martínez Z, Iñiguez LC, Rodríguez T. 1997. Influence of effects on quality traits and relationships between traits of the llama fleece. Small Ruminant Res 24: 203-212. doi: 10.1016/S0921-4488(96)00925-X

22. McGregor B. 2006. Production attributes and relative value of alpaca fleeces in Southern Australia and implications for industry development. Small Ruminant Res 61: 93-111. doi: 10.1016/j.smallrumres.2005.07.001

23. McGregor B. 2012. Properties, processing and performance of rare natural animal fibres: a review and interpretation of existing research results. Melbourne: Rural Industries Research and Development Corporation, Australia. [Internet]. Disponible en: https://www.researchgate.net/publication/306259308_Properties_processing_an-d_performance_of_rare_and_natural-_fibres_a-_review_and_interpretation_of_existing_research_results.

24. McGregor B. 2013. Medullated fibres in mohair. Mohair news. [Internet]. Disponible en: https://dro.deakin.edu.au/ eserv/DU:30062590/mcgregor medullatedfibres-2013.pdf.

25. McGregor B, Quispe EC. 2018. Cuticle and cortical cell morphology of alpaca and other rare animal fibres. J Text I 109: 767-774. doi: 10.1080/00405000.2017.1368112

26. Montes M, Quicaño I, Quispe R, Quispe E, Alfonso L. 2008. Quality characteristics of Huacaya alpaca fibre produced in the Peruvian Andean Plateau region of Huancavelica. Span J Agric Res 6:33-38. doi: 10.5424/sjar/2008061-5258

27. Moore K. 2015. The impact of fleece characteristics on insulation and heat exchange, and the consequential effect on vitamin D of alpacas in southern Australia. PhD Thesis. Perth: University of Western Australia. $152 \mathrm{p}$.

28. Omachea E, Calsín B, Olarte U. 2015. Características textiles de la fibra en alpacas Huacaya del distrito de Corani, Carabaya, Puno. Rev Invest Altoandin 17: 215-220.

29. Onal L, Korkmaz M, Tutak M. 2007. Relations between the characteristics of Angora rabbit fibre. Fiber Polym 8: 198204. doi: 10.1007/BF02875792

30. Pinares R, Gutiérrez G, Cruz A, Morante R, Cervantes I, Burgos L, Gutiérrez JP. 2018. Heritability of individual fiber medullation in Peruvian alpacas. Small Ruminant Res 165: 93100. doi: 10.1016/j.smallrumres.2018.04.007

31. Pinares R, Gutiérrez G, Cruz A, Burgos L, Gutiérrez JP. 2019. Variabilidad fenotípica del porcentaje de fibras meduladas en el vellón de alpacas Huacaya. Rev Inv Vet Perú 30: 699-708. doi: 10.15381/rivep.v30i2.16098

32. Poma GF. 2018. Evaluación de las características físicas de la fibra de llama (Lama glama) a la primera esquila en la mancomunidad de municipios Aymaras sin fronteras para su procesamiento en la industria textil. Apthapi 4: 1275-1286.

33. Quispe EC, Alfonso L, Flores A, Guillen H, Ramos Y. 2009. Bases para un programa de mejora de alpacas en la región altoandina de Huancavelica-Perú. Arch Zootec 58: 705-716. doi: 10.4321/ S0004-05922009000400008

34. Radzik A, Chopra S, Lonkar P. 1995. Study of wool quality and skin follicles in some of the typical Indian sheep breeds. Annals Warsaw Agric Univ Anim Sci 31: 41-46. 
35. Radzik A, Pofelska O, Rant W. 2018. Characteristics of alpaca wool from farmed animals located on different continents. SGGW Anim Sci 57: 151-158. doi: 10.22630/AAS.2018.57.2.15

36. Rafat $\mathrm{SA}$, de Rochambeau $\mathrm{H}$, Brims $M$, Thébault $G$, Deretz, $S$, Bonnet $M$, Allain D. 2007. Characteristics of Angora rabbit fiber using optical fiber diameter analyser. J Anim Sci 85: 3116 3122. doi: $10.2527 /$ jas.2007-0109

37. R Core Team. 2019. R: A language and environment for statistical computing. $\mathrm{R}$ Foundation for Statistical Computing, Vienna, Austria. https://www.R-project.-org/

38. Shakyawar DB, Kadam V, Suya AK, Ahmed A, Pareek PK, Temani P. 2013. Precise mesurement of wool fibre diameter using computerized projection microscope. Ind J Small Ruminant 19: 190-192.

39. Süpüren G, Özdil N, Özçelik G 2014. Physical properties of angora rabbit fibers. Am J Mater Eng Tech 2: 11-13. doi: 10.12691/materials-2-2-2
40. Vásquez D, Perovic P, de Olsen A. 2000. Patrones cuticulares y medulares de pelos de mamíferos del noroeste argentino (Carnivora y Artiodactyla). Mastozool Neotrop 7: 131-147.

41. Vasquez R, Gómez E, Quispe EC. 2015. Características tecnológicas de la fibra blanca de alpaca huaca en la zona altoandina de Apurímac. Rev Inv Vet Perú 26: 213-222. doi: 10.15381/ rivep.v26i2.11020

42. Wang X, Wang L, Liu X. 2003. The quality and processing performance of alpaca fibres. Geelong: Rural Industries Research and Develpment Corporation. Publication No. 03/128, [Internet]. Disponible en: https://www.agrifutures.com.au/wp-content/uploads/publications/ 03-128.pdf

43. Wang H., Liu X, Wang X. 2005. Internal structure and pigment granules in colored alpaca fibers. Fiber Polym 6: 263-268. doi: 10.1007/BF02875652 